\title{
Internações por traumatismo cranioencefálico em Sergipe, de 2000 a 2011
}

\author{
Natália de Jesus Viana ${ }^{1}$, Anna Klara Bohland², Carlos Umberto Pereira ${ }^{3}$
}

Universidade Federal de Sergipe (UFS), Aracaju, SE, Brasil.

\section{RESUMO}

Objetivo: Avaliar o perfil epidemiológico das vítimas de traumatismo cranioencefálico (TCE) que tiveram internação hospitalar em instituições cobertas pelo Sistema Único de Saúde - SUS, de 2000 a 2011. Método: Pesquisa descritiva com abordagem quantitativa. Utilizaram-se dados secundários provenientes do Sistema de Informações Hospitalares (SIH-SUS). Para o estudo, foram utilizados: taxa de internações por TCE por idade, sexo, ano, mês e duração da internação, óbitos hospitalares, diagnóstico secundário e valor da internação. Para fins de análise, foi utilizado o programa Tabwin. Resultados: O coeficiente de hospitalização em Sergipe variou de 22,8 a 38,1 por 100.000 habitantes e o coeficiente de mortalidade hospitalar foi de 4,5 por 100.000 habitantes por ano. As internações concentraram-se em homens (82,2\%) e entre 20 a 39 anos (43,3\%). A maior causa de internação e óbitos foram as causas cuja intenção é indeterminada (36,6\% das internações e 42,4\% dos óbitos), seguidas pelos acidentes de trânsito (33,2\% e 32\%, respectivamente). A letalidade hospitalar foi de $13,7 \%$, sendo as agressões físicas e a faixa etária idosa as circunstâncias mais relacionadas ao óbito hospitalar. Conclusões: É necessário conhecer perfil da população acometida para traçar estratégias individuais e coletivas de prevenção desses agravos. O uso dos bancos de dados disponíveis é uma ferramenta valiosa nesse contexto.

\section{PALAVRAS-CHAVE}

Traumatismos craniocerebrais, hospitalização, letalidade.

\section{ABSTRACT}

Hospitalization for traumatic brain injury in Sergipe, of 2000 to 2011

Objective: To evaluate the epidemiology of traumatic brain injury (TBI) who had hospitalization in institutions covered by the Unified Health System - SUS, 2000-2011. Method: Descriptive research with quantitative approach. We used secondary data from the Hospital Information System (SIH-SUS). For this study, were used: rate of hospitalizations for TBI by age, sex, year, month and duration of stay, in-hospital deaths, secondary diagnosis and value of hospitalization. For purposes of analysis Tabwin was used. Results: The coefficient of hospitalization in Sergipe ranged from 22.8 to 38.1 per 100,000 inhabitants in the period and the Coefficient of in-hospital Mortality in period was 4.5 per 100,000 inhabitants per year. The hospitalizations were most on men (82.2\%) and between 20 and 39 years (43.3\%). The leading cause of hospitalization and death were the causes of indeterminate intent (36.6\% of hospitalizations and $42.4 \%$ of deaths), followed by traffic accidents (33.2\% and $32 \%$, respectively). Hospital lethality was $13.7 \%$, with assaults and elderly age group as the circumstances most related to hospital death. Conclusions: It is necessary to know the profile of the affected population to trace individual and collective strategies for prevention of these diseases. The use of available databases is a valuable tool in this context.

\section{KEYWORDS}

Craniocerebral trauma, hospitalization, lethality.

1 Médica-residente do Serviço de Radiologia e Diagnóstico por Imagem do Hospital Universitário Pedro Ernesto, Universidade Estadual do Rio de Janeiro (UERJ), Rio de Janeiro, RJ, Brasil.

2 Professora do Departamento de Medicina da Universidade Federal de Sergipe (UFS), Aracaju, SE, Brasil.

3 Professor do Departamento de Medicina da UFS, Aracaju, SE, Brasil. 


\section{INTRODUÇÃO}

O trauma é responsável por mais de 5,8 milhões de mortes a cada ano, representando $10 \%$ da mortalidade mundial. ${ }^{1}$ No Brasil, as causas externas são a principal causa de óbito entre 1 e 39 anos. $^{2}$ De acordo com a OMS, o trauma, especialmente o neurotrauma, será responsável pela maior morbidade e mortalidade no mundo por volta do ano $20200^{3,4}$

O traumatismo cranioencefálico (TCE) é definido como qualquer lesão de origem traumática que provoque lesão ou déficit funcional do couro cabeludo, crânio, meninges, encéfalo ou seus vasos, sendo considerado a lesão de maior morbidade e mortalidade. Estima-se que esse agravo seja responsável por cerca de um terço das mortes relacionadas ao trauma. ${ }^{5}$ Dos politraumatizados que falecem, ele é a causa em $33 \%$ a $50 \%$ dos casos. Do total de mortes por acidentes de trânsito, $60 \%$ são causados primariamente pelo TCE. ${ }^{6}$

A incidência mundial é estimada em 106 casos para cada 100.000 habitantes. Porém, esses dados variam de acordo com a região que se estuda. Em países desenvolvidos, a incidência é estimada em 200 casos por 100.000 habitantes por ano, sendo geralmente usados para essas estimativas dados de internações hospitalares. ${ }^{5}$ De acordo com o Centers for Disease Control and Prevention (CDC), nos Estados Unidos, estima-se que 1,7 milhão de pessoas por ano sofrem um TCE, das quais 52 mil morrem, 275 mil são hospitalizadas e 80\% (1.365 milhões) são atendidas e liberadas na emergência. ${ }^{7}$ No Brasil, de 2001 a 2007, o TCE foi responsável por uma média anual de 640.686 internações hospitalares, com crescimento de aproximadamente $24 \%$ no período. ${ }^{8}$ Apesar disso, o neurotrauma recebe suporte insuficiente das entidades governamentais e privadas, sendo chamado de epidemia silenciosa por alguns autores. ${ }^{4}$

Há dificuldade na realização de estudos comparativos sobre o tema, pois, além de serem escassos, ainda se encontram problemas de ordem metodológica na obtenção dos dados, uma vez que os diagnósticos e critérios de inclusão nesses estudos não são uniformes, com análises comparativas muito difíceis de serem feitas. ${ }^{9}$ Alguns valem-se apenas dos registros de internação hospitalar, outros também incluem visitas a departamentos de emergências e eventos fatais extra-hospitalares, conseguindo contabilizar das formas mais brandas às mais graves do TCE. ${ }^{5}$

Tratando-se as causas externas de eventos passíveis de prevenção por ações individuais e coletivas, tornase necessária a caracterização de perfis de morbidade e mortalidade, assim como a melhor caracterização das vítimas de violência, para criação de políticas preventivas efetivas. Fazer isso por meio da análise de banco de dados disponíveis no Brasil não é uma tarefa fácil e, ao mesmo tempo, é importante por apresentar não somente as possibilidades, mas também as dificuldades e limitações dessas ferramentas, servindo para indicar as falhas e, portanto, sugerir melhorias.

Dentro dessa perspectiva, é proposto um estudo que caracterize as internações por TCE no Sistema Único de Saúde (SUS) ocorridas no Estado de Sergipe de acordo com o perfil epidemiológico e as circunstâncias que causaram a lesão, por meio de uma ferramenta epidemiológica valiosa, que é o Sistema de Informações Hospitalares do Sistema Único de Saúde (SIH/SUS).

\section{Método}

Trata-se de uma pesquisa exploratória com uma abordagem quantitativa que permitiu identificar o perfil das vítimas hospitalizadas por TCE, no estado de Sergipe, a partir do SIH-SUS.

Foram analisadas as internações hospitalares do período de 2000 a 2011. Para tanto, foram utilizados dados secundários, selecionados com base na lista de morbidade da Classificação Estatística Internacional de Doenças e Problemas Relacionados à Saúde (OMS, 1995), 10 ${ }^{\text {a }}$ Revisão (CID-10). ${ }^{10}$ Foram utilizadas as seguintes subcategorias:

- Fratura de crânio e ossos faciais (S02), em que foram inclusas a fratura da abóbada de crânio (S02.0), a fratura da base do crânio (S02.1), as fraturas múltiplas envolvendo crânio e ossos faciais (S07.7), as fraturas de crânio e outros ossos faciais (S02.8) e fratura do crânio ou dos ossos da face, parte não especificada (S02.9).

- Ferimento intracraniano (S06), em que foram inclusas todas as subcategorias.

- Lesão por esmagamento da cabeça (S07), em que foram inclusas apenas as subcategorias lesão por esmagamento do crânio (S07.1) e traumatismo por esmagamento da cabeça, parte não especificada (S07.9).

Os dados de internação hospitalar e respectivo desfecho (óbito ou não óbito), bem como os dados populacionais, estão disponíveis no site do Ministério da Saúde. ${ }^{11}$

Para o estudo, foram utilizados os seguintes indicadores: taxas brutas de internações por TCE, em Aracaju, de 2000 a 2011, por idade, sexo, ano, mês e duração da internação hospitalar, óbitos hospitalares, diagnóstico secundário da internação, e valor da internação. Para fins de análise, foi utilizado o programa TabWin. ${ }^{12}$

Os diagnósticos secundários das internações foram agrupados em grupos de causa, que mostram a circunstância em que ocorreu o acidente. Foram excluídos 
desse agrupamento 12 pacientes que apresentavam essa informação não preenchida.

Para cálculo de coeficientes de morbidade em Sergipe, foram excluídos atendimentos a pacientes residentes de outras Unidades da Federação. Portanto, os dados foram mínimos, uma vez que só foram utilizados os casos ocorridos em Sergipe e residentes do Estado.

O presente projeto foi aprovado pelo Comitê de Ética em Pesquisa em 4/2/2013 (CAAE: 11539012.0.0000.5546).

\section{Resultados}

Foi registrado um total de 8.978 atendimentos hospitalares por TCE em Sergipe, de 2000 a 2011, sendo 7.683 (85,5\%) residentes no Estado. Em 2000, a população em Sergipe era de 1.784.475 habitantes e, em 2011, de 2.089.819 habitantes, apresentando um crescimento de $17,1 \%$. O número de hospitalizações por TCE no ano de 2000 foi de 669 e em 2011 foi de 851, com média de 748 casos por ano, apresentando aumento de $27,2 \%$ no período.

Quando considerados apenas os residentes de Sergipe, o número de hospitalizações por TCE no ano de 2000 foi de 562 e em 2011 foi de 752, com média anual de 640 casos, apresentando aumento de 33,8\%. O coeficiente de morbidade hospitalar variou de $22,8 / 100.000$ a 38,1/100.000 habitantes. Como se observa na tabela 1 , os coeficientes de internação apresentavam uma pequena tendência crescente até o ano de 2007. Em 2008, apresentaram uma queda e alcançaram seus menores valores, retornando aos patamares anteriores nos anos seguintes. Cabe frisar que esses coeficientes de morbidade hospitalar são mínimos, pois somente foram utilizados os casos de residentes e ocorridos no estado de Sergipe.

Observando os coeficientes de internação por causa, nota-se que a tendência dos acidentes de trânsito seguiu a tendência geral, crescendo até 2007, apresentando diminuição brusca em 2008. O coeficiente de internação por quedas apresentou diminuição ao longo do tempo, assim como o das agressões. Ao verificar os coeficientes dos eventos cuja intenção foi indeterminada, esses apresentaram tendência crescente ao longo do período, principalmente em 2010 e 2011.

Ainda na tabela 1 , pode-se constatar que o coeficiente de morbidade hospitalar foi predominante no sexo masculino em todos os anos. A relação sexo masculino:sexo feminino variou de 3,7:1 até 5,5:1. A menor diferença entre os riscos masculino e feminino é encontrada nas faixas etárias menores de cinco anos, com o sexo feminino chegando a superar o masculino em alguns anos. A partir dessa idade, há aumento pro- gressivo na internação por TCE no sexo masculino, chegando a 11,2 vezes mais chance de um homem de 20 a 39 anos ser internado por TCE no estado de Sergipe do que uma mulher na mesma faixa etária. Essa diferença diminui na faixa etária dos maiores de 60 anos, por aumento no coeficiente do sexo feminino.

Quando se consideram os coeficientes de morbidade por faixa etária, pode-se observar que as maiores frequências ocorreram nas faixas etárias de 20 a 39 anos e nos maiores de 60 anos. A faixa etária de 40 a 59 anos também apresentou coeficientes elevados, aparecendo como a segunda maior em alguns anos da série. A faixa etária dos menores de um ano, apesar de não se manter constantemente elevada, apresentou aumentos importantes, como nos anos de 2003, 2007 e 2009.

Quando consideradas todas as internações, independente do local de residência, a caracterização das vítimas de acordo com a causa do trauma (Tabela 2) mostrou que a maior parte dos diagnósticos secundários apresentou eventos cuja intenção é indeterminada como circunstância do TCE $(36,6 \%)$, seguidos pelos acidentes de transporte $(33,2 \%)$ e quedas $(18,8 \%)$. O sexo masculino predominou em todas as causas, alcançando valores de até mais que $90,0 \%$, como nas agressões e nas lesões autoprovocadas.

Do total das internações, $82,2 \%$ corresponderam ao sexo masculino (Tabela 2). Quanto à faixa etária, a de maior proporção foi de 20 a 39 anos, correspondendo a $43,3 \%$ do total, seguida da faixa de 40 a 59 anos e 10 a 19 anos (19,3\% e 14,4\%, respectivamente).

Quanto às principais causas de TCE de acordo com a idade (Figura 1), os eventos cuja intenção é indeterminada representaram grande parcela em todas as faixas etárias estudadas, chegando a ser a maior parte em menores de um ano, entre 40 e 59 anos e maiores de 60 anos. Excluindo esses eventos, as quedas predominaram nas faixas etárias menores de 10 anos (35,7\%, 34,1\% e $34,7 \%$ das internações em menores de um ano, crianças de 1 a 4 anos e de 5 a 10 anos, respectivamente). Entre 10 e 39 anos, os acidentes de transporte predominaram, alcançando $43,3 \%$ na faixa etária de 20 a 39 anos $(39,4 \%$ entre 10 e 19 anos). Nos idosos, chama a atenção o fato de que as agressões foram a maior causa de internação por TCE, com $23,9 \%$, seguidas pelas quedas $(18,6 \%)$, quando se excluem os eventos cuja intenção é indeterminada.

De acordo com os dias de permanência (Tabela 3), a maior parte das internações foi entre zero e sete dias. Os custos com essas internações representaram 37,0\% do custo total com internações por TCE. Ao longo do período, o número de internações com sete dias ou menos diminuiu, enquanto as mais longas aumentaram. Os custos também aumentaram, com média de R $\$ 1.154,20$ por internação e custo dia de R \$ 188,70.

As características dos óbitos hospitalares podem ser vistas na tabela 4 . Foram registrados 1.231 óbitos hospitalares, sendo 1.060 de residentes em Sergipe. 


\begin{tabular}{|c|c|c|c|c|c|c|c|c|c|c|c|c|}
\hline \multirow[t]{2}{*}{ Coeficiente } & \multicolumn{12}{|c|}{ Ano de internação } \\
\hline & 2000 & 2001 & 2002 & 2003 & 2004 & 2005 & 2006 & 2007 & 2008 & 2009 & 2010 & 2011 \\
\hline \multicolumn{13}{|l|}{ Causa } \\
\hline Acidentes de transporte & 8,1 & 6,8 & 12,1 & 13,0 & 13,3 & 12,6 & 13,7 & 16,0 & 7,8 & 8,1 & 7,4 & 8,4 \\
\hline Quedas & 9,4 & 6,9 & 8,5 & 9,2 & 8,5 & 7,7 & 7,0 & 4,0 & 3,3 & 5,1 & 3,5 & 3,6 \\
\hline $\begin{array}{l}\text { Exposição a forças } \\
\text { mecânicas inanimadas }\end{array}$ & 0,1 & 0,4 & 0,9 & 1,3 & 0,7 & 1,3 & 0,9 & 0,1 & 0,1 & - & - & - \\
\hline $\begin{array}{l}\text { Exposição a forças } \\
\text { mecânicas animadas }\end{array}$ & 0,1 & 0,1 & 0,0 & 0,1 & 0,1 & 0,2 & 0,2 & - & - & - & - & - \\
\hline Lesões autoprovocadas & 0,6 & 0,4 & 0,0 & 0,2 & 0,2 & 0,4 & 1,2 & 0,2 & 0,0 & 0,0 & 0,0 & 0,0 \\
\hline Agressões & 2,6 & 1,3 & 2,4 & 3,4 & 3,2 & 2,4 & 1,7 & 1,6 & 0,9 & 1,6 & 1,3 & 1,7 \\
\hline $\begin{array}{l}\text { Eventos com intenção } \\
\text { indeterminada }\end{array}$ & 9,9 & 8,7 & 10,7 & 10,7 & 7,7 & 9,2 & 8,4 & 12,8 & 9,9 & 10,9 & 22,0 & 22,1 \\
\hline $\begin{array}{l}\text { Complicações de } \\
\text { assistência médica }\end{array}$ & 0,4 & 0,4 & 0,9 & 0,1 & 0,1 & 0,1 & - & - & - & - & - & - \\
\hline Outros & 0,2 & 0,3 & 0,2 & 0,2 & 0,6 & 0,2 & 3,1 & 3,0 & 0,5 & - & - & - \\
\hline Total & 31,5 & 25,4 & 37,1 & 38,1 & 34,2 & 34,0 & 36,5 & 37,9 & 22,8 & 25,9 & 34,3 & 36,0 \\
\hline \multicolumn{13}{|l|}{ Faixa etária } \\
\hline$<1$ ano & 36,0 & 20,2 & 27,3 & 39,1 & 12,0 & 30,2 & 22,9 & 44,3 & 19,5 & 41,9 & 30,9 & 39,7 \\
\hline $1-4$ anos & 20,9 & 16,8 & 21,4 & 27,7 & 23,1 & 12,6 & 15,2 & 19,9 & 9,7 & 20,3 & 13,2 & 29,7 \\
\hline $5-9$ anos & 15,4 & 14,6 & 26,8 & 16,6 & 24,6 & 20,5 & 20,6 & 18,6 & 12,0 & 16,9 & 10,6 & 20,5 \\
\hline $10-19$ anos & 22,7 & 15,8 & 25,5 & 23,0 & 22,0 & 19,7 & 24,0 & 28,1 & 16,5 & 17,4 & 18,5 & 25,4 \\
\hline $20-39$ anos & 41,1 & 37,3 & 48,3 & 51,4 & 46,3 & 47,6 & 50,5 & 48,1 & 25,8 & 28,1 & 47,5 & 42,5 \\
\hline $40-59$ anos & 36,5 & 25,0 & 41,9 & 49,5 & 36,7 & 41,0 & 46,5 & 38,1 & 30,4 & 27,4 & 38,6 & 39,4 \\
\hline 60 anos e mais & 41,2 & 32,3 & 51,0 & 46,7 & 48,2 & 51,7 & 46,1 & 58,8 & 35,1 & 43,4 & 48,4 & 45,8 \\
\hline Total & 31,5 & 25,4 & 37,1 & 38,1 & 34,2 & 34,0 & 36,5 & 37,9 & 22,8 & 25,9 & 34,3 & 36,0 \\
\hline \multicolumn{13}{|l|}{ Sexo masculino } \\
\hline$<1$ ano & 45,2 & 14,8 & 38,8 & 38,2 & 18,8 & 31,8 & 35,7 & 59,5 & 28,7 & 48,4 & 42,6 & 42,2 \\
\hline $1-4$ anos & 22,5 & 19,6 & 30,1 & 27,3 & 26,8 & 14,7 & 15,5 & 17,3 & 10,8 & 21,7 & 14,4 & 38,4 \\
\hline $5-9$ anos & 13,5 & 11,9 & 25,4 & 12,2 & 22,0 & 23,8 & 23,4 & 19,8 & 13,7 & 17,9 & 12,2 & 17,7 \\
\hline $10-19$ anos & 38,4 & 25,9 & 43,3 & 34,6 & 33,1 & 32,0 & 39,0 & 37,9 & 22,5 & 26,6 & 33,5 & 47,8 \\
\hline $20-39$ anos & 74,9 & 67,6 & 86,9 & 91,7 & 83,3 & 88,3 & 91,9 & 85,2 & 48,2 & 49,9 & 87,1 & 78,7 \\
\hline $40-59$ anos & 57,7 & 47,9 & 81,2 & 87,2 & 71,8 & 77,0 & 78,5 & 67,2 & 56,2 & 49,1 & 66,4 & 68,7 \\
\hline 60 anos e mais & 70,7 & 57,7 & 90,3 & 72,6 & 79,8 & 80,6 & 74,8 & 95,5 & 52,0 & 66,3 & 86,7 & 84,7 \\
\hline Total & 51,4 & 42,6 & 63,7 & 60,5 & 56,8 & 58,5 & 61,2 & 61,5 & 38,6 & 42,0 & 58,8 & 61,1 \\
\hline \multicolumn{13}{|l|}{ Sexo feminino } \\
\hline$<1$ ano & 26,4 & 25,9 & 15,3 & 40,1 & 4,9 & 28,6 & 9,4 & 28,5 & 9,9 & 35,2 & 18,8 & 37,2 \\
\hline $1-4$ anos & 19,3 & 13,9 & 12,4 & 28,1 & 19,3 & 10,5 & 14,9 & 22,6 & 8,7 & 18,7 & 11,9 & 20,7 \\
\hline $5-9$ anos & 11,5 & 12,3 & 17,2 & 15,9 & 17,6 & 6,6 & 7,4 & 8,0 & 4,1 & 8,1 & 4,5 & 16,9 \\
\hline $10-19$ anos & 7,9 & 6,3 & 8,6 & 12,2 & 11,5 & 8,0 & 9,7 & 15,3 & 8,5 & 5,9 & 8,4 & 9,7 \\
\hline $20-39$ anos & 9,2 & 8,7 & 11,8 & 13,2 & 11,4 & 9,2 & 11,5 & 12,5 & 4,3 & 7,2 & 10,4 & 8,6 \\
\hline $40-59$ anos & 17,1 & 4,0 & 6,0 & 15,0 & 4,5 & 8,1 & 17,2 & 11,9 & 7,2 & 8,0 & 13,7 & 13,1 \\
\hline 60 anos e mais & 17,8 & 12,1 & 19,9 & 26,2 & 23,2 & 28,8 & 23,4 & 29,6 & 21,7 & 25,2 & 18,3 & 15,2 \\
\hline Total & 12,3 & 8,9 & 11,5 & 16,4 & 12,5 & 10,6 & 12,7 & 15,1 & 7,6 & 10,3 & 11,2 & 12,3 \\
\hline
\end{tabular}

Fonte: Datasus, 2013. ${ }^{*}$ Foram excluídos 12 casos por não apresentarem preenchimento do campo diagnóstico secundário. 
Tabela 2 - Distribuição em número e percentual dos pacientes internados por TCE, por faixa etária e causa ${ }^{\star}$ do trauma segundo o sexo. Sergipe, de 2000 a 2011

\begin{tabular}{|c|c|c|c|c|c|c|}
\hline \multirow[t]{2}{*}{ Características das vítimas } & \multicolumn{2}{|c|}{ Homens } & \multicolumn{2}{|c|}{ Mulheres } & \multicolumn{2}{|c|}{ Total } \\
\hline & $\mathbf{N}$ & $\%$ & $\mathbf{N}$ & $\%$ & $\mathbf{N}$ & $\%$ \\
\hline \multicolumn{7}{|l|}{ Faixa etária } \\
\hline$<1$ ano & 96 & 62,3 & 58 & 37,7 & 154 & 1,7 \\
\hline $1-4$ anos & 240 & 58,1 & 173 & 41,9 & 413 & 4,6 \\
\hline $5-9$ anos & 360 & 71,4 & 144 & 28,6 & 504 & 5,6 \\
\hline $10-19$ anos & 1019 & 78,8 & 274 & 21,2 & 1293 & 14,4 \\
\hline $20-39$ anos & 3451 & 88,7 & 440 & 11,3 & 3891 & 43,3 \\
\hline $40-59$ anos & 1486 & 85,6 & 249 & 14,4 & 1735 & 19,3 \\
\hline 60 anos e mais & 732 & 74,1 & 256 & 25,9 & 988 & 11,0 \\
\hline \multicolumn{7}{|l|}{ Causas do trauma } \\
\hline Evento cuja intenção é indeterminada & 2662 & 81,2 & 617 & 18,8 & 3279 & 36,6 \\
\hline Acidentes de transporte & 2488 & 83,5 & 493 & 16,5 & 2981 & 33,2 \\
\hline Agressões & 496 & 91,7 & 45 & 8,3 & 541 & 6,0 \\
\hline Quedas & 1319 & 78,2 & 367 & 21,8 & 1686 & 18,8 \\
\hline Lesões autoprovocadas & 71 & 92,2 & 6 & 7,8 & 77 & 0,9 \\
\hline Exposição a forças mecânicas inanimadas & 122 & 88,4 & 16 & 11,6 & 138 & 1,5 \\
\hline Exposição a forças mecânicas animadas & 16 & 88,9 & 2 & 11,1 & 18 & 0,2 \\
\hline Outras & 200 & 81,3 & 46 & 18,7 & 246 & 2,7 \\
\hline Total $^{*}$ & 7.374 & 100,0 & 1.592 & 100,0 & 8.966 & 100,0 \\
\hline
\end{tabular}

* Foram excluídos 12 casos por não apresentarem preenchimento do campo diagnóstico secundário.

Fonte: Datasus, 2013.

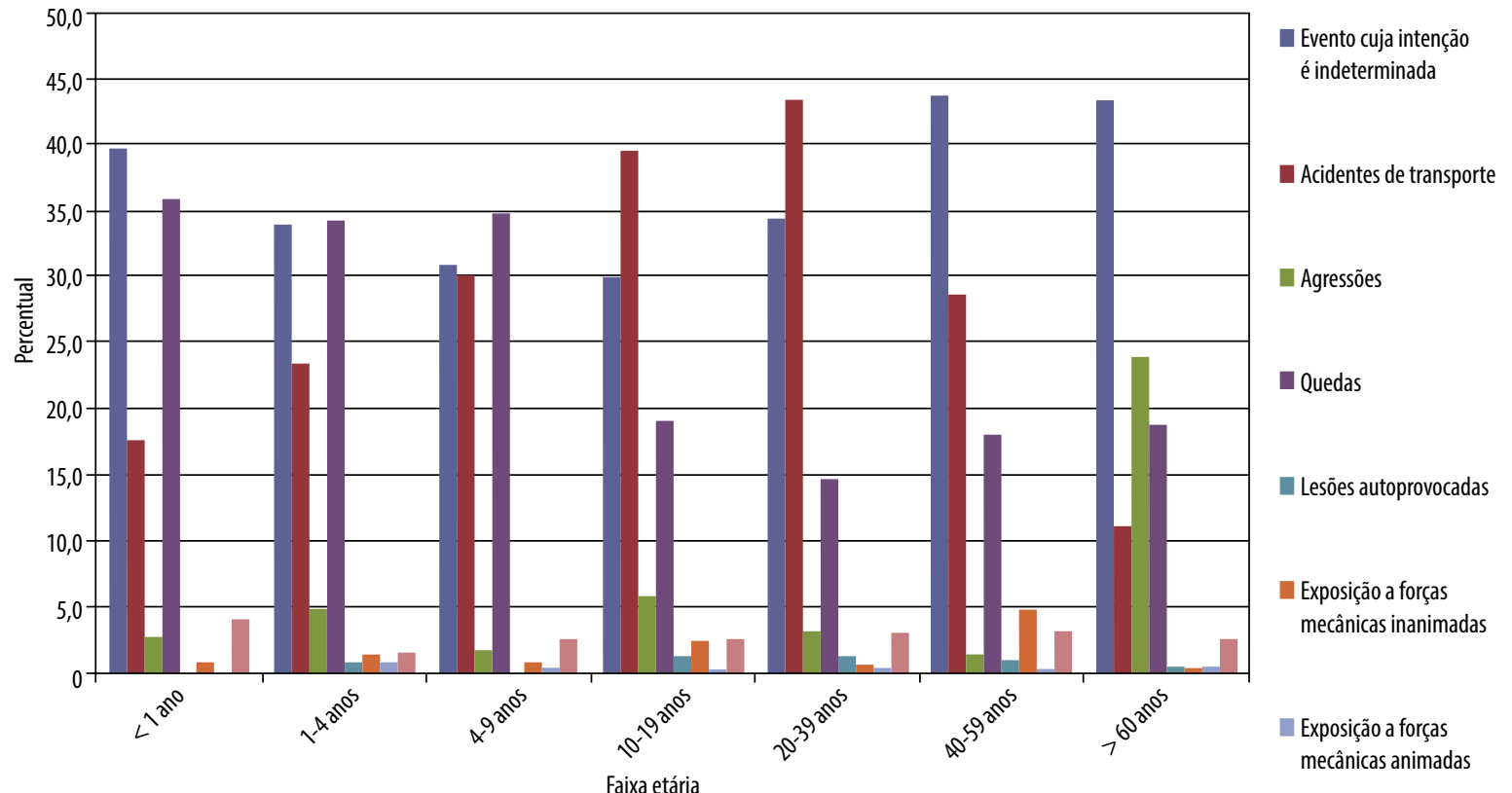

Figura 1 - Distribuição percentual de causas ${ }^{\star}$ de acordo com a faixa etária dos pacientes internados por TCE no Estado de Sergipe, de 2000 a 2011.

* Foram excluídos 12 casos por não apresentarem preenchimento do campo diagnóstico secundário. 


\begin{tabular}{|c|c|c|c|c|c|c|c|c|c|c|c|c|}
\hline & \multicolumn{12}{|c|}{ Ano } \\
\hline & 2000 & 2001 & 2002 & 2003 & 2004 & 2005 & 2006 & 2007 & 2008 & 2009 & 2010 & 2011 \\
\hline Coeficiente & 4,5 & 3,7 & 4,4 & 4,4 & 4,7 & 5 & 4,7 & 4,8 & 2,4 & 4,9 & 5,4 & 5,2 \\
\hline \multicolumn{13}{|l|}{ Letalidade } \\
\hline $0-7$ dias & 17,0 & 16,2 & 14,4 & 13,6 & 17,4 & 16,4 & 15,5 & 15,8 & 11,7 & 23,0 & 22,1 & 19,9 \\
\hline $8-14$ dias & 10,5 & 9,4 & 6,6 & 3,7 & 8,2 & 9 & 8,1 & 6,2 & 11,8 & 17,5 & 7,5 & 12,2 \\
\hline $15-21$ dias & 4,9 & 4,3 & 2,3 & 7 & 5,6 & 2,9 & 7,5 & 10,3 & 9,4 & 11,9 & 10,1 & 3,9 \\
\hline $22-28$ dias & 10,5 & - & 14,3 & 5,9 & 21,1 & 10,5 & - & 10,3 & - & 3,3 & 3,8 & 6,5 \\
\hline 29 dias e mais & 8,7 & 7,1 & 9,5 & 14,3 & 10,3 & 19,4 & 14,3 & 5 & 3,3 & 10,7 & 8,9 & 5,8 \\
\hline Total & 14,5 & 14,1 & 12,1 & 11,2 & 14,5 & 14,3 & 12,8 & 12,5 & 10,6 & 18,5 & 15,4 & 14,7 \\
\hline \multicolumn{13}{|c|}{ \% Dias de permanência } \\
\hline $0-7$ dias & 67,7 & 75,8 & 70,9 & 70,0 & 66,2 & 69,0 & 62,1 & 62,5 & 62,1 & 54,3 & 52,2 & 54,3 \\
\hline $8-14$ dias & 19,9 & 15,6 & 19,2 & 19,0 & 20,6 & 19,5 & 21,8 & 21,3 & 22,2 & 20,7 & 25,7 & 21,3 \\
\hline $15-21$ dias & 6,1 & 4,2 & 5,5 & 5,0 & 7,0 & 4,7 & 6,2 & 6,4 & 6,0 & 11,0 & 9,5 & 8,9 \\
\hline $22-28$ dias & 2,8 & 1,8 & 1,8 & 2,0 & 2,5 & 2,6 & 3,3 & 3,2 & 4,1 & 4,9 & 3,1 & 5,4 \\
\hline 29 dias e mais & 3,4 & 2,6 & 2,6 & 4,1 & 3,8 & 4,2 & 6,6 & 6,6 & 5,6 & 9,2 & 9,5 & 10,1 \\
\hline
\end{tabular}

Fonte: Datasus, 2013.

\begin{tabular}{|c|c|c|c|c|c|c|}
\hline \multicolumn{7}{|c|}{$\begin{array}{c}\text { Tabela } 4 \text { - Distribuição em número e percentual dos casos de óbitos entre os pacientes internados por } \\
\text { TCE, por faixa etária e causa do trauma segundo o sexo*. Sergipe, de } 2000 \text { a } 2011\end{array}$} \\
\hline \multirow[t]{2}{*}{ Características das vítimas } & \multicolumn{2}{|c|}{ Homens } & \multicolumn{2}{|c|}{ Mulheres } & \multicolumn{2}{|c|}{ Total } \\
\hline & $\mathbf{N}$ & $\%$ & $\mathbf{N}$ & $\%$ & $\mathbf{N}$ & $\%$ \\
\hline \multicolumn{7}{|l|}{ Faixa etária } \\
\hline$<1$ ano & 5 & 0,5 & 2 & 1,0 & 7 & 0,6 \\
\hline $1-4$ anos & 12 & 1,2 & 4 & 2,0 & 16 & 1,3 \\
\hline $5-9$ anos & 14 & 1,4 & 8 & 4,1 & 22 & 1,8 \\
\hline $10-19$ anos & 114 & 11,0 & 14 & 7,1 & 128 & 10,4 \\
\hline $20-39$ anos & 477 & 46,1 & 40 & 20,3 & 517 & 42,0 \\
\hline $40-59$ anos & 243 & 23,5 & 48 & 24,4 & 291 & 23,6 \\
\hline$>60$ anos & 169 & 16,3 & 81 & 41,1 & 250 & 20,3 \\
\hline \multicolumn{7}{|l|}{ Causas do trauma } \\
\hline Acidentes de transporte & 349 & 33,8 & 45 & 22,8 & 394 & 32 \\
\hline Quedas & 118 & 11,4 & 24 & 12,2 & 142 & 11,5 \\
\hline Exposição a forças mecânicas inanimadas & 19 & 1,8 & 2 & 1,0 & 21 & 1,7 \\
\hline Exposição a forças mecânicas animadas & 1 & 0,1 & - & - & 1 & 0,1 \\
\hline Lesões autoprovocadas & 10 & 1,0 & - & - & 10 & 0,8 \\
\hline Agressões & 100 & 9,7 & 8 & 4,1 & 108 & 8,8 \\
\hline Evento cuja intenção é indeterminada & 411 & 39,7 & 111 & 56,3 & 522 & 42,4 \\
\hline Outras & 25 & 2,4 & 7 & 3,6 & 32 & 2,6 \\
\hline Total $^{*}$ & 1.034 & 100,0 & 197 & 100,0 & $1.230^{*}$ & 100,0 \\
\hline
\end{tabular}

Fonte: Dataus, 2013

* Um caso de óbito masculino foi excluído dos cálculos de óbito por causa, por não apresentar o diagnóstico secundário 
O coeficiente de mortalidade hospitalar do TCE foi de 4,5 óbitos para cada 100.000 habitantes por ano no período. Seu menor valor foi em 2008, com 2,4 óbitos para cada 100.000 habitantes e o maior foi em 2010, quando alcançou 5,4 óbitos/100.000 (Tabela 3). Ressalta-se que os dados desse coeficiente são mínimos, por serem compostos apenas pelos óbitos ocorridos em Sergipe.

Considerados todos os óbitos hospitalares ocorridos, a taxa de letalidade hospitalar do estudo foi de $13,7 \%$. Quanto às circunstâncias, a maior parte dos óbitos foi causada por eventos cuja intenção é indeterminada, seguida pelos acidentes de transporte $(42,4 \%$ e $32 \%$, respectivamente), sendo esse perfil observado em ambos os sexos.

Quanto à idade, predominou a faixa de 20 a 39 anos, com $42 \%$ de todos os óbitos. A letalidade por faixa etária foi crescente à medida que a idade avançava, a partir dos cinco anos de idade, alcançando seu maior valor entre os maiores de 60 anos $(25,3 \%)$. A maior letalidade por causa observada foi no grupo das agressões, com 20,0\%, seguida de eventos cuja intenção é indeterminada, exposição a forças mecânicas inanimadas e acidentes de transporte (15,9\%, 15,2\% e 13,2\%, respectivamente).

Considerando todos os pacientes internados por TCE em Sergipe, a taxa de letalidade de acordo com os dias de permanência foi maior nas internações entre zero e sete dias (Tabela 3), que contribuíram com $65,8 \%$ do total de óbitos e apresentaram um crescimento ao longo dos anos. Esse aumento nas taxas de óbito também ocorreu com as internações de 8 a 14 dias. A letalidade das internações de 15 a 21 dias e de 22 a 28 dias oscilou, mas se estabeleceram taxas menores ao final do período. Nas internações de mais de 29 dias tiveram tendência decrescente a partir de 2006.

\section{Discussão}

A tendência crescente do TCE em Sergipe acompanhou a tendência nacional e internacional. Estudo semelhante utilizando os dados do SIH-SUS mostrou um crescimento do número de TCE no Brasil de $24 \%$ de 2001 a $2007^{8}$ e, nos Estados Unidos, o CDC constatou aumento de $34 \%$ nas hospitalizações de 2002 a 2006. ${ }^{7}$

O coeficiente de morbidade hospitalar dos residentes em Sergipe no período estudado permaneceu abaixo do coeficiente brasileiro encontrado no estudo de Fernandes ${ }^{8}$, que variou de 39 a 56 casos por 100.000 habitantes. Koizume et al. ${ }^{9}$ encontraram o valor de $36 / 100.000$ habitantes em estudo semelhante ao nosso para determinar a morbimortalidade no município de São Paulo. Esses coeficientes encontram-se abaixo dos encontrados na Europa (235/100.000 por ano $)^{13} \mathrm{e}$ em outros países desenvolvidos, que ficam em torno de 200/100.000 habitantes. ${ }^{5}$

A comparação entre os coeficientes de morbimortalidade estimados torna-se difícil pela grande variação dos critérios de inclusão e bancos de dados analisados. Enquanto alguns incluem mortes extra-hospitalares e atendimentos em emergência sem hospitalização, outros incluem apenas hospitalizações, subestimando o real número dos TCE. ${ }^{5,9}$ Os coeficientes de países desenvolvidos podem ser maiores simplesmente por apresentarem um sistema de informação mais desenvolvido e capaz de registrar maior número de ocorrências, ou por apresentarem melhor serviço de atendimento a urgências pré-hospitalares, evitando óbitos na cena ou a caminho do hospital.

Considerando todas as internações do período, quanto às circunstâncias destas, excluindo os eventos com intenções indeterminadas, os acidentes de trânsito foram os responsáveis pelas maiores taxas durante o período e também apresentaram os maiores coeficientes de internação por TCE entre 2002 e 2007. Nos Estados Unidos, as quedas são a principal causa de hospitalizações $(35,2 \%)$, enquanto os acidentes de transporte são a principal causa de morte entre esses atendimentos $(31,8 \%)^{7}$

Em diversos estudos nacionais e internacionais, os acidentes de trânsito se mantiveram entre as principais causas de morbidade por causas externas, sendo a primeira causa em diversos deles. ${ }^{5,13-18}$ Há a previsão de que em 2030 os acidentes de trânsito serão a quinta causa de morte em todo o mundo. ${ }^{1}$ Além do grande número de ocorrências, cerca de 400 mil pessoas tornam-se inválidas e 33 mil pessoas morrem anualmente devido a esses acidentes. Os índices de mortalidade viária no Brasil são altos, sendo uma das principais causas de mortalidade prematura. ${ }^{14}$

Nos anos de 2008 e 2009 ocorreu um fenômeno nos dados de morbidade no trânsito no Brasil que também foi observado em nosso estudo. Em 2008, houve declínio do número de internações em uma série histórica sobre acidentes de trânsito no Brasil, seguido de aumento de mais de 30\% em 2009. Questiona-se se isso seria um efeito da Lei $\mathrm{n}^{\circ}$ 11.705/08 (Lei Seca) implantada em 2008, ou erro no sistema de informação que poderia ter influenciado tamanha variação. ${ }^{19} \mathrm{Em} 2008$, houve queda no coeficiente de internação por acidentes de trânsito em Sergipe. Ao mesmo tempo, nenhuma internação por TCE menor que um dia foi registrada no SIH em Sergipe neste ano, fato que pode ter contribuído para o declínio das internações nesse ano, sendo um fator de confusão na interpretação dos resultados.

As agressões representaram apenas 6\% das internações em nosso estudo, com coeficientes de internação não tão altos. Esse perfil também foi encontrado em outro estudo brasileiro, em que as agressões foram 
responsáveis pela menor proporção de hospitalizações por TCE, fato que pode ser explicado pela alta mortalidade no local da agressão, perdendo a contribuição para os dados de internação e mortalidade hospitalar, além da omissão dos fatos pela própria vítima. ${ }^{8}$

A predominância do sexo masculino é corroborada na literatura médica, seja se tratando de TCE, quanto de lesões por causas externas em geral. $5,8,9,14,15,18,20-23$ Eles representam a grande parcela dos envolvidos em violências e acidentes. Acredita-se que essa diferença ocorra pelo fato de que os homens são mais expostos desde crianças às causas externas, por apresentar mais independência, entrarem mais cedo no mercado de trabalho e adotarem comportamento de risco, como álcool e direção. Um estudo realizado em Minas Gerais mostrou que, entre os pacientes atendidos com TCE que apresentavam níveis elevados de alcoolemia, mais de $90 \%$ eram do sexo masculino. ${ }^{14}$

Esse predomínio geralmente é mantido em todas as faixas etárias, alcançando a maior relação sexo masculino:sexo feminino entre os adolescentes e adultos jovens. ${ }^{5}$ No presente estudo, essa relação variou de 3,7:1 a 5,5:1. Em estudo de Pereira et al., ${ }^{24}$ essa relação foi de 2,7:1, enquanto em estudo realizado por Moura et al..$^{22}$ essa relação chegou a 6,2:1.

De acordo com a literatura médica, existem três picos de incidência de TCE: crianças pequenas, final da adolescência/adultos jovens e idosos representam os grupos mais vulneráveis. ${ }^{5}$ Em grande parte dos estudos nacionais, assim como no presente trabalho, os adultos jovens são os responsáveis pela maior parte das internações por causas externas., ${ }^{8,14,15,17,20,22}$ As menores incidências observadas em adultos de meia-idade são uma tendência quase universal, precedendo o último pico de incidência em idosos, ${ }^{5}$ fato que não ocorreu em nosso estudo, pois esses foram representantes da segunda maior frequência de internações e de elevados coeficientes de morbidade hospitalar.

As crianças, apesar de não representarem grande porcentagem das internações no presente estudo, contribuíram com um aumento de incidência entre os menores de um ano nos anos de 2003, 2007 e 2009. Elas representam uma grande parcela das vítimas de TCE, representando o primeiro pico de acordo com a faixa etária. ${ }^{5}$ Nos Estados Unidos, menores de quatro anos representam a maior parte dos atendimentos em emergências devido a TCE, cuja principal causa são as quedas. ${ }^{7}$ As crianças apresentam uma faixa etária vulnerável a traumas, pois apresentam pouco discernimento sobre situações perigosas, como, por exemplo, no trânsito. ${ }^{25}$

As quedas predominaram como causa na faixa etária até os 10 anos (ao excetuar as causas cuja intenção é indeterminada), fato que é corroborado pela literatura médica. ${ }^{23,25,26}$ As circunstâncias em que ocorrem essas quedas variam de acordo com a faixa etária, predominando as quedas de berço e colo de mãe entre os lactentes, quedas de escadas em período escolar e quedas relacionadas a lazer ou meio de transportes (bicicleta, esportes) em pré-adolescentes. ${ }^{23}$ Os meninos são mais vulneráveis que as meninas também nessa faixa etária, podendo ser explicado pelo fato de o menino adquirir mais liberdade e mais precocemente que a menina. ${ }^{23}$ Pela dependência de cuidadores nessa faixa etária, deve-se chamar a atenção tanto para a necessidade de políticas de prevenção voltadas a estes, que devem repassar hábitos seguros para os menores, quanto para o fato dos maus-tratos e negligência infantil. ${ }^{1,27,28}$

$\mathrm{O}$ aumento da expectativa de vida faz surgir uma nova preocupação em relação aos idosos e sua vulnerabilidade a acidentes e violências. Em nosso estudo, o coeficiente de internação por TCE em idosos variou de 32,3 a 58,8 por 100.000 habitantes acima de 60 anos. Os fatores que mais contribuem nacionalmente para a mortalidade por causas externas em idosos são os considerados acidentais, com os acidentes de trânsito (os maiores responsáveis) e quedas somando cerca de $42,7 \% .{ }^{29}$ Outros estudos também apontam as quedas e acidentes de trânsito como maiores causas de internação e óbito em idosos. ${ }^{5}$

Diferente do resultado encontrado no presente estudo, as agressões representam pequena porcentagem nas internações entre os mais velhos. Em estudo de Pinheiro et al. , $^{30}$ as quedas foram responsáveis por $48,8 \%$ das internações por TCE em idosos, enquanto acidentes de trânsito representam $31,7 \%$ e as agressões, 7,3\%. Nos Estados Unidos, as agressões foram responsáveis por apenas $1 \%$ das hospitalizações em maiores de 65 anos. ${ }^{7}$ Os grandes índices de maus-tratos aos idosos, a dependência social e econômica, a institucionalização e a vulnerabilidade dessa população podem contribuir para os altos índices de violência contra essa faixa etária encontrados em Sergipe durante o período. De acordo com Malta et al., ${ }^{31}$ a grande maioria dessas agressões ocorre no próprio lar, partindo de filhos, cônjuges, genros/noras, muitas vezes usuários de drogas. A violência doméstica é responsável por $90 \%$ das agressões nessa faixa etária. ${ }^{31}$

De acordo com a duração da internação, a grande maioria ocorreu no grupo de 0-7 dias. As internações por causas externas têm a característica de apresentarem internações menos duradouras e mais custosas (19\% a mais dos custos no Brasil) que as causas naturais. ${ }^{32}$ Durante o período, os custos com internações de 0 a 7 dias representaram aproximadamente $37 \%$ do custo total com internações por TCE. O custo médio por internação foi de $\mathrm{R} \$ 1.154,20$ por paciente, muito acima do encontrado em estudo de Melione e Mello-Jorge, ${ }^{33}$ que foi de $\mathrm{R} \$ 684,82$. O custo dia também foi mais elevado 
que foi encontrado nesse estudo, sendo de $\mathrm{R} \$ 188,70$, em comparação com $\mathrm{R} \$ 112,93$ do estudo anterior.

Vale ressaltar que tais custos apresentados são apenas os considerados custos primários, que são os representados pelos custos médicos e não médicos de diagnóstico, tratamento e reabilitação, não mensurando os custos indiretos, como a perda de dias de trabalho, e limitações físicas que prejudiquem a produtividade. ${ }^{32}$ O aumento nas taxas de internação com altos gastos pode ser causa e consequência de maior investimento em tratamentos de alta complexidade e reabilitação de vítimas de TCE em vez de investir em políticas de prevenção.

Quando considerados todos os óbitos ocorridos, as taxas de letalidade hospitalar foram crescentes no presente estudo, principalmente a letalidade hospitalar precoce (0 a 7 dias). O Sistema de Informação sobre Mortalidade do Ministério da Saúde (SIM/MS) mostra que o Brasil vem apresentando aumento no número de mortes por causas externas, seja em taxas de mortalidade proporcional, seja em relação aos seus coeficientes. ${ }^{34}$ É importante frisar que estudos que utilizam a Autorização de Internação Hospitalar (AIH) como fonte apresentam limitações devido ao fato de contabilizar somente os óbitos hospitalares de pacientes atendidos pelo Sistema Único de Saúde, deixando os óbitos que ocorrem na cena de fora dos cálculos.

A letalidade hospitalar encontrada por Koizumi et al. ${ }^{9}$ e Ramos et al..$^{35}$ foi de 10,1\% (município de São Paulo) e de $10,5 \%$ (município de Caruaru), respectivamente, semelhante ao nosso estudo, enquanto Melo et al. ${ }^{20}$ encontraram a taxa de 22,9\% (município de Salvador). Em estudos realizados na Europa, as taxas de letalidade hospitalar ficaram em torno de $3,0 \%$, enquanto a taxa de letalidade geral foi de $11,0 \%{ }^{13}$ Isso mostra melhor atendimento hospitalar na Europa, pois nossas taxas de letalidade hospitalar são comparáveis às taxas gerais de lá, que incluem, além dos óbitos hospitalares, vítimas que foram a óbito sem atendimento médico.

Quanto às causas de óbito, os acidentes de trânsito representaram a maioria no presente estudo, ao excluir os eventos cuja intenção foi indeterminada. No Brasil, eles representam cerca de $28,0 \%$ do total de óbitos por causas externas, com 35 mil mortes por ano e 95 mortes por dia. Em Sergipe, de 2000 a 2010 houve aumento de $77,7 \%$ nos óbitos por acidentes de trânsito, ${ }^{36}$ não sendo encontrado esse crescimento em nossos resultados. Houve queda desses óbitos em determinadas faixas etárias a partir do ano de 1998, ano da implementação do Código de Trânsito Brasileiro. ${ }^{37-39}$ Até 1997, era crescente o número de mortes no trânsito registrado pelo SIM. No entanto, essa queda nos óbitos somente durou até 2000 , quando voltou a crescer cerca de $4,8 \%$ ao ano, retornando aos patamares de 1997 em 2005 e continuando a crescer de forma sistemática, tendendo a ultrapassar a maior causa de óbitos violentos: os homicídios. ${ }^{36}$
Na literatura médica predominam os casos de atropelamento de pedestres, estando a proporção aumentada quando se consideram menores de 15 anos e idosos. ${ }^{34}$ De 1996 a 2010, a mortalidade dos pedestres diminuiu $51,5 \%$ no Brasil, porém, todas as outras categorias sofreram acréscimo, especialmente a dos motociclistas, com crescimento de aproximadamente $846 \%$ no mesmo período. Se retirássemos os motociclistas das contas, a mortalidade por acidentes de trânsito haveria diminuído entre 1996 e 2010 e não aumentado, mostrando que nos dias atuais esta é a categoria que mais demanda medidas de controle da violência no trânsito. ${ }^{36}$

As agressões, apesar de não representarem a maior porcentagem de internações, apresentaram a maior letalidade no estudo. O perfil de mortalidade também diferiu do perfil de internação no estudo de Koizumi et al., ${ }^{9} \mathrm{em}$ que as agressões foram a terceira causa de internação, mas representaram a maior proporção de mortalidade, e no estudo de Asemota et al. ${ }^{16} \mathrm{em}$ que as armas de fogo foram o mecanismo mais letal entre as causas de TCE em adolescentes.

Os números em relação à violência são alarmantes. Em estudo de Fernandes, ${ }^{8}$ as agressões foram o fator mais fortemente associado ao óbito entre as causas analisadas. Todo ano, somente a violência vitima fatalmente cerca de um milhão e meio de pessoas, e muitas outras ficam feridas e sofrem com as sequelas, em idades jovens, muitas vezes perdendo para sempre sua capacidade laborativa e diminuindo sobremaneira sua qualidade de vida. ${ }^{27}$ As agressões são maiores no sexo masculino em todas as faixas etárias. ${ }^{37} \mathrm{~A}$ estimativa de morrer por homicídio no sexo masculino é 10 vezes maior que no sexo feminino. ${ }^{34}$

Chama a atenção a grande proporção de eventos cuja intenção é indeterminada encontrada em Sergipe, tanto nas causas de internamento quanto de óbito. Esse diagnóstico secundário apresentou tendência crescente, pondo em cheque a qualidade dos dados de $\mathrm{AIH}$ no Estado. A falta de treinamento na emissão e preenchimento das AIH é responsável por esses altos índices, tornando difícil sua padronização e o uso de dados para comparações entre diferentes regiões ${ }^{40}$ Problemas como esse levam a estudos como o de Cerqueira, ${ }^{41}$ que observou uma tendência à queda do número de homicídios enquanto houve aumento de mortes violentas cuja intenção não foi esclarecida a partir de 2007. Ao se aprofundar nessas mortes, foi constatado que $62,5 \%$ dos casos registrados correspondiam a homicídios ocultos.

As causas mal definidas de mortalidade apresentaram queda entre 1980 e 2008 no Brasil, sendo observada uma redução percentual de $65 \%$. O Nordeste, região que apresentava as maiores taxas de mortes mal definidas, apresentou uma redução de $83 \%$ nesse período, sendo mais pronunciada a partir de $2004 .{ }^{42}$ Apesar disso, estudos que analisam mortalidade por causas violentas no 
Brasil frisam que o Nordeste apresenta grande número de óbitos por causas mal definidas além do sub-registro, o que leva a uma subnotificação que pode mascarar o verdadeiro risco de morte e alterar o perfil epidemiológico das causas externas. ${ }^{37,43}$ Esse fato transparece no presente estudo, com altos índices de morbimortalidade por eventos cuja intenção é indeterminada.

Levando em conta todos os óbitos, a faixa etária predominante em Sergipe foi a dos adultos jovens, fato que reflete o perfil nacional de óbitos por TCE. ${ }^{8}$ Ao se calcular a letalidade por faixa etária, os idosos apresentaram a maior taxa de letalidade hospitalar. Os idosos representam um dos picos de incidência do TCE e, apesar de não corresponder à maioria das internações na literatura médica e em nosso estudo, estes apresentam as maiores taxas de letalidade hospitalar e coeficientes de mortalidade, até três vezes maiores que da população em geral. ${ }^{7,29}$

Ao longo do período, as internações com duração de 0 a 7 dias diminuíram proporcionalmente, enquanto a letalidade nessas internações aumentou. Poderia ser inferido que a maior mortalidade precoce dessas vítimas devido a TCEs mais graves estaria diminuindo a duração das internações. Já o aumento proporcional das internações mais longas e a diminuição nas taxas de letalidade hospitalar destas poderiam se relacionar a um melhor atendimento hospitalar, com novas tecnologias e formas de tratamento para essas vítimas, além do maior prolongamento das vidas de pacientes com TCE grave e sequelas elevarem o número de dias de internação e gastos com métodos diagnósticos e de cuidado.

\section{Conclusão}

A abordagem ao problema das causas externas atualmente ultrapassa o âmbito apenas curativo da lesão traumática e coloca como prioridade a prevenção desses eventos. Além da organização dos serviços móveis de urgência, centros de atendimento e de reabilitação, o enfoque preventivo deve ser reforçado, assim como o papel da atenção básica na educação coletiva para promoção da saúde e prevenção de agravos.

Os sistemas de informação são tidos como a ferramenta mais importante no controle de qualidade do atendimento ao trauma. Além de dados referentes ao agravo, esse registro deve conter informações demográficas e epidemiológicas, servindo para a análise dos casos de forma individual e coletiva. O desenvolvimento de intervenções tem como base o conhecimento em nível local do perfil da população acometida.

Como dito anteriormente, a dificuldade em comparar diversos estudos sobre TCE reside não só no fato das grandes variações nos critérios de inclusão ou na escolha do banco de dados, mas também nas diferentes abordagens dadas ao problema. Estudos de séries temporais sobre o assunto, avaliando a variação dos coeficientes de morbidade por sexo, faixa etária ou causa ao longo dos anos, são raros, não sendo encontrado nenhum na literatura médica brasileira, sendo mais fácil a obtenção de dados percentuais relativos às internações do que medidas de risco.

Os dados referentes a Sergipe coincidem com a literatura médica em geral, em que homens e jovens são as principais vítimas do TCE, com a maior parcela relacionada a acidentes de trânsito. A faixa etária dos idosos apresentou um padrão diferente do encontrado na literatura, alertando para a necessidade de uma nova abordagem de proteção aos idosos no Estado. A tendência crescente no número de internações segue um padrão mundial, em que as causas externas ganham cada vez mais espaço na sociedade e chamam a atenção para a necessidade de esforços na prevenção desses agravos.

Além disso, sabe-se que uma parcela considerável de eventos externos, especialmente a violência interpessoal entre grupos vulneráveis, não chega ao conhecimento das autoridades competentes, necessitando constantemente de campanhas de conscientização e proteção dessas vítimas, muitas vezes necessitando de respaldo legal. O caráter multifacetado da violência e dos acidentes está cada vez mais claro para as autoridades e sociedade, demandando de atenção multidisciplinar e multissetorial.

Pode-se concluir que os bancos de morbidade são ferramentas valiosas para o estudo das causas externas, permitindo a obtenção do perfil das vítimas e de suas circunstâncias. Por depender de recursos humanos, torna-se subjetivo e sujeito a erros. O elevado número de diagnósticos secundários incompletos ou inexistentes mostra a desvantagem na utilização desse banco de dados. A conscientização sobre a importância e o maior treinamento dos profissionais de saúde ou até mesmo a criação de protocolos para preenchimento dos dados nos locais de internação são importantes para que estes se tornem mais confiáveis, sejam mais utilizados como fonte de informação e tornem-se mais significativos para a análise da situação de saúde da localidade.

Mesmo com essas limitações, é possível traçar um perfil epidemiológico, capaz de direcionar políticas preventivas individuais e coletivas e que auxiliem também na avaliação dessas condutas.

\section{Conflitos de interesse}

Os autores declaram não haver conflitos de interesse. 


\section{Referências}

1. World Health Organization (WHO). Injuries and violence: the facts; 2010. Disponível em: http://www.who.int/violence injury_prevention/key_facts/en/. Acesso em: 21 Ago, 2014.

2. Malta DC, Silva MMA, Barbosa J. Violências e acidentes, um desafio ao Sistema Único de Saúde. Ciênc Saúde Coletiva. 2012;17(9):2220.

3. Hyder AA, Wunderlich CA, Puvanachandra P, Gururaj G, Kobusingye OC. The impact of traumatic brain injuries: a global perspective. NeuroRehabilitation. 2007;22(5):341-53.

4. Zitnay GA. Lessons from national and international TBI societies and fundslike NBIRTT. Acta Neurochir Suppl. 2005;93:131-3.

5. Bruns J Jr, Hauser WA. The epidemiology of traumatic brain injury: a review. Epilepsia. 2003;44 Suppl 10:2-10.

6. Guzmán F. Fisiopatología del trauma craneoencefálico. Colomb Med. 2008;39(Suppl 3):78-84.

7. Centers for Disease Control and Prevention. Traumatic Brain Injury in The United States: Emergency Departments Visits, Hospitalizations and Deaths 2002-2006; 2010. Disponível em: http://www.cdc.gov/traumaticbraininjury/ pdf/blue_book.pdf. Acesso em: 4 Set, 2014

8. Fernandes RNR. Análise Epidemiológica das Hospitalizações no Sistema Único de Saúde, por Traumatismo Crânio Encefálico. Brasil: 2001-2007 [dissertação]. Salvador: Universidade Federal da Bahia; 2010.

9. Koizumi MS, Lebrão ML, Mello-Jorge MH, Primerano V. Morbidity and mortality due to traumatic brain injury in São Paulo City, Brazil, 1997. Arq Neuropsiquiatr. 2000;58(1):81-9.

10. Organização Mundial da Saúde. Classificação estatística internacional de doenças e problemas relacionados à saúde: $10^{a}$ rev. 1995. São Paulo: Centro Colaborador da OMS para Classificação de Doenças em Português. Universidade de São Paulo; 1995.

11. Brasil, Ministério da Saúde. Informações de Saúde; 2012. Disponivel em: http://www2.datasus.gov.br/DATASUS/ index.php?area=02. Acesso em: 7 Jan, 2013.

12. Brasil. Ministério da Saúde. Tabwin. Disponível em: http://www2.datasus.gov.br/DATASUS/index. php?area=040805\&item=3. Acesso em: 7 Jan 7, 2013.

13. Tagliaferri F, Compagnone C, Korsic M, Servadei F, Kraus J. A systematicr eview of brain injury epidemiology in Europe. Acta Neurochir (Wien). 2006;148(3):255-68.

14. Faria JW, Nishioka Sde A, Arbex GL, Alarcão GG, Freitas WB. Occurrence of severe and moderate traumatic brain injury in patients attended in a Brazilian Teaching Hospital: epidemiology and dosage of alcoholemy. Arq Neuropsiquiatr. 2008;66(1):69-73.

15. Ruy EL, Rosa MI. Perfil epidemiológico de pacientes com traumatismo crânio encefálico. ACM Arq Catarin Med. 2011;40(3):17-22.

16. Asemota AO, George BP, Bowman SM, Haider AH, Schneider EB. Causes and trends in traumatic brain injury for United States adolescents. J Neurotrauma. 2013;30(2):67-75

17. Barbosa IL, Andrade LM, Caetano JA, Lima MA, Vieria LJES, Lira SVG. Fatores desencadeantes ao trauma crânioencefálico em um Hospital de Emergência Municipal. Rev Baiana Saúde Pública. 2010;34(2):240-53.

18. Nunes MS, Hora EC, Fakhouri R, Alves JAB, Ribeiro MCO, Santos ACFS. Caracterização de vítimas de Traumatismo Atendidas em um Hospital de Urgência. Rev Enferm UFPE on line. 2011;5(9):2136-42. Disponível em: 10.5205/ reuol.1262-12560-1-LE.0509201109. Acesso em: 29 Ago, 2013.
19. Bacchieri G, Barros AJD. Acidentes de trânsito no Brasil de 1998 a 2010: muitas mudanças e poucos resultados. Rev Saúde Pública. 2011;45(5):949-63.

20. Melo JR, Silva RA, Moreira ED Jr. Characteristics of patients with head injury at Salvador City (Bahia--Brazil). Arq Neuropsiquiatr. 2004;62(3A):711-4.

21. Maia BG, Paula FRP, Cotta GD, Cota MAL, Públio $P G$, Oliveira $H$, et al. Perfil clínico-epidemiológico das ocorrências de traumatismo cranioencefálico. Rev Neurociênc. 2013;21(1):43-52.

22. Moura JC, Rangel BLR, Creônio SCE, Pernambuco JRB. Perfil clínico-epidemiológico de traumatismo cranioencefálico do Hospital de Urgências e Traumas no município de Petrolina, estado de Pernambuco. Arq Bras Neurocir. 2011;30(3):99-104.

23. Machado Filho JA, Silva AC, Machado MMT, Madureira RA, Carvalho FHA, Santiago LR, et al. Perfil clínicoepidemiológico das crianças e adolescentes hospitalizados por traumatismo crânio encefálico. Rev Bras Promo Saúde. 2010;23(4):335-42.

24. Pereira CU, Duarte GC, Santos EAS. Avaliação epidemiológica do traumatismo craniencefálico no interior do Estado de Sergipe. Arq Bras Neurocir. 2006;25(1):8-16.

25. Melo JRT, Santana DLP, Pereira JLB, Ribeiro TF. Traumatismo craniencefálico em crianças e adolescentes na cidade do Salvador - Bahia. Arq Neuropsiquiatr. 2006;64(4):994-6.

26. Pereira CU, Lima AA. Vômitos em traumatismo cranioencefálico leve na infância. Arq Bras Neurocir. 2013;32(2):59-62

27. World Health Organization (WHO). Violence prevention: the evidence; 2010. Disponível em: http://www.who.int/ violence_injury_prevention/violence/4th_milestones_ meeting/publications/en/. Acesso em: $21 \mathrm{Abr}, 2013$.

28. Ebehard FME, Pose G, Mora X, Vergara MF. Maltrato infantil, TEC y diagnóstico por imágenes. Rev Chil Pediatr. 2011;82(3):231-7.

29. Gawryszewski VP, de Mello Jorge MH, Koizumi MS. Injury among the elderly: thechallenge to integrate preventive activities in public and individual levels. Rev Assoc Med Bras. 2004;50(1):97-103.

30. Pinheiro Al, De Almeida FM, Barbosa IV, Mesquita Melo E, Borges Sturdat RM, De Figueiro Carvalho ZM. Principais causas associadas ao traumatismo cranioencefálico em idosos. Eferm Glob. 2011;10(12):1-11.

31. Malta DC, Lemos MSA, Silva MMA, Rodrigues EMS, Gazal-Carvalho C, Morais Neto OL. Iniciativas de vigilância e prevenção de acidentes e violência no contexto do Sistema Único de Saúde (SUS). Epidemiol Serv Saúde. 2007;16(1):45-55.

32. Mello-Jorge MHP, Koizumi MS. Gastos governamentais do SUS com internações hospitalares por causas externas: análise no Estado de São Paulo, 2000. Rev Bras Epidemiol. 2004;7(2):228-38.

33. Melione LP, Mello-Jorge MH. Unified National Health System costs in São José dos Campos, São Paulo State, Brazil, for hospital admissions due to external causes. Cad Saude Publica. 2008;24(8):1814-24.

34. Mello-Jorge MHP, Koizumi MS, Tono VL. Causas externas. O que são, como afetam o setor saúde, sua medida e alguns subsídios para sua prevenção. Rev Saúde. 2007;1(1):37-47.

35. Ramos EMS, Silva MKB, Siqueira GR, Vieira RAG, França WLC. Aspectos epidemiológicos dos traumatismos cranioencefálicos atendidos no Hospital Regional do Agreste de Pernambuco de 2006 a 2007. Rev Bras Promo Saúde. 2010;23(1):4-10.

36. Waiselfisz JJ, Instituto Sangari. Mapa da Violência 2012. Caderno Complementar 2: Acidentes de Trânsito; 2012. 
Disponível em URL: http://mapadaviolencia.org.br/pdf2012/ mapa2012_transito.pdf. Acesso em: 13 Set, 2013.

37. Brasil. Secretaria de Vigilância em Saúde. Ministério da Saúde. Saúde Brasil 2004 - Uma análise da situação de saúde; 2004. Disponível em: http://portal.saude.gov.br/portal/arquivos/pdf/ saude_brasil_2004.pdf. Acesso em: 14 Set, 2013.

38. Brasil. Secretaria de Vigilância em Saúde. Ministério da Saúde. Saúde Brasil 2005 - Uma análise da situação de saúde; 2005. Disponível em: http://bvsms.saude.gov.br/ bvs/publicacoes/saude_brasil_2005parte3.pdf. Acesso em: 14 Set, 2013.

39. Brasil. Secretaria de Vigilância em Saúde. Ministério da Saúde. Mortalidade por acidentes de transporte terrestre no Brasil; 2007. Disponível em: http://www.prosaude. org/publicacoes/diversos/livro_mortalidade_transito.pdf. Acesso em: 14 Set, 2013.

40. Lemos C, Chaves LDP, Azevedo ALCS. Sistemas de informação hospitalar no âmbito do SUS: revisão integrativa de pesquisas. Rev Eletrônica Enferm. 2010;12(1):177-85.
41. Cerqueira D. Mortes violentas não esclarecidas e impunidade no Rio de Janeiro. Economia Aplicada. 2012;16(2):201-35.

42. Brasil. Secretaria de Vigilância em Saúde. Ministério da Saúde. Saúde Brasil 2009: Uma análise da situação de saúde e da agenda nacional e internacional de prioridades em saúde; 2009. Disponível em: http://portalsaude. saude.gov.br/portalsaude/arquivos/pdf/2013/Fev/28/ saudebrasil2009_parte1_cap2.pdf. Acesso em: 7 Out, 2013.

43. Paes NA. Qualidade nas estatísticas de óbitos por causas desconhecidas dos Estados brasileiros. Rev Saúde Pública. 2007;41(3):436-45.

\section{Endereço para correspondência}

Natália de Jesus Viana

Av. Alan Kardec, 660, ap. 103

Bairro Cirurgia

49055-030 - Aracaju, SE, Brasil

E-mail: njviana@gmail.com 